\title{
DENSIDADES DE ARANHAS ERRANTES (CTENIDAE E SPARASSIDAE, ARANEAE) EM UMA FLORESTA FRAGMENTADA
}

\author{
Felipe N. A. A. Rego ${ }^{1,2}$, Eduardo M. Venticinque ${ }^{1,2,3}$ \& Antonio D. Brescovit ${ }^{4}$ \\ Biota Neotropica v5 (n1a) -http://www.biotaneotropica.org.br/v5n1a/pt/abstract?article+BN002051a2005 \\ Recebido em 07/12/2003 \\ Aceito em 01/02/2005 \\ ${ }^{1}$ Curso de Pós-graduação em Ecologia, Instituto Nacional de Pesquisas da Amazônia (INPA). Caixa Postal 478, \\ CEP: 69011-970, Manaus, AM, Brasil. http://www.inpa.gov.br/ pgeco/ \\ E-mail: regofelipe@yahoo.com \\ 2Projeto Dinâmica Biológica de Fragmentos Florestais (PDBFF), Caixa Postal 478, CEP 69011-970, Manaus, AM. Brasil. \\ http://pdbff.inpa.gov.br/ \\ ${ }^{3}$ Wildlife Conservation Society (WCS), Amazon-Andes Conservation Program. Rua dos Jatobás, 274, Coroado 3. \\ CEP 69085-000, Manaus, AM, Brasil. http://www.wcs.org/international/latinamerica/amazon andes \\ E-mail: eventicinque@wcs.org \\ ${ }^{4}$ Laboratório de Artrópodes Peçonhentos, Instituto Butantan, Av. Vital Brasil, 1500, São Paulo, 05530-900, Brasil. \\ E-mail: anyphaenidae@butantan.gov.br
}

\begin{abstract}
The wandering spiders of the families Ctenidae and Sparassidae are nocturnal and can be found mainly on the leaf litter, and in sites close to each other in the Amazon. The coexistence of these spiders suggests that they compete for food, shelters and, as a generalist predator, the intraguild predation can also limit the abundance of their populations. In this study, we investigated the forest fragmentation effects on the density of spiders, whose populations are under the influence of isolation and reduction of their habitats. The densities of Ctenidae and Sparassidae species in seven forest fragments ( $\leq 10 \mathrm{ha}$ ) and nine areas of continuous forest (>1000 ha) in a "terra-firme" (non-flooded) forest of Central Amazon were compared. ANOVA tests revealed that ctenid spiders are more susceptible to the forest fragmentation by having their populations reduced in fragments where, on the other hand, sparassid spiders were more abundant. Therefore, the studied fragments were considered to be disturbed environments since they are not capable of maintaining these spider populations at the same levels as that observed in continuous forest. Besides, the negative correlation between the density of ctenid and sparassid spiders in the study area (Pearson, $r=-0,527 ; n=16$ ) indicates that, probably the competition for resources and perhaps, the intraguild predation are important factors for the size of these populations. Nevertheless, a better knowledge on the interactions between Ctenidae and Sparassidae species (interspecific competition and intraguild predation) is fundamental to understand how the coexistence between them influences the susceptibility of these spiders to the forest fragmentation.
\end{abstract}

Key words: wandering spiders; Ctenidae; Sparassidae; habitat fragmentation; Central Amazon.

Resumo - As aranhas errantes das famílias Ctenidae e Sparassidae são noturnas e podem ser encontradas principalmente no folhiço forrageando em locais próximos umas das outras na região amazônica. A coexistência entre estas aranhas sugere que elas competem por alimento e abrigos e, por serem predadores generalistas, a predação intraguilda também pode limitar a abundância de suas populações. Neste estudo, investigamos os efeitos da fragmentação florestal sobre a densidade de aranhas, cujas populações estão sujeitas ao isolamento e a redução de seus hábitats. Sete fragmentos florestais ( $\leq 10$ ha) e nove áreas de mata contínua (>1000 ha) foram comparados quanto à densidade das espécies de Ctenidae e Sparassidae, em uma floresta de terra-firme na Amazônia Central. Testes com ANOVA revelaram que os ctenídeos foram mais suscetíveis à fragmentação da floresta por terem suas populações reduzidas nos fragmentos onde, por outro lado, os esparassídeos foram mais abundantes. Portanto, os fragmentos estudados foram considerados ambientes alterados por não serem capazes de manter as populações destas aranhas nos níveis observados na mata contínua. Além disso, a correlação entre a densidade de ctenídeos e esparassídeos foi negativa na área de estudo (Pearson, $r=-0,527, n=16$ ) indicando que é provável, que a competição por recursos e, talvez, a predação intraguilda tenham um papel importante no tamanho destas populações. Porém, um melhor conhecimento a respeito das interações entre as espécies de Ctenidae e Sparassidae (competição interespecífica e predação intraguilda) é fundamental para o entendimento de como a coexistência entre elas pode influenciar na suscetibilidade destas aranhas à fragmentação da floresta.

Palavras-chave: aranhas errantes; Ctenidae; Sparassidae; fragmentação de hábitats; Amazônia Central. 


\section{INTRODUÇÃO}

Atividades humanas como a pecuária, agricultura, exploração de madeira e a construção de estradas são as principais causas do aumento das taxas de desmatamento na Amazônia durante a última década (Laurance et. al. 2002). A derrubada e queima da floresta contribuem para o surgimento de fragmentos florestais, que são remanescentes de vegetação nativa isolados e circundados por uma paisagem alterada pela ação antrópica (Davidson 1998). Nos fragmentos, o isolamento e a redução da floresta alteram a estrutura da vegetação (Didham \& Lawton 1999), o microclima (Camargo \& Kapos 1995) e, conseqüentemente, o hábitat de muitos invertebrados (Didham 1997a). Estas alterações na paisagem influenciam a composição de espécies de formigas de liteira (Carvalho \& Vasconcelos 1999) e explicam a menor abundância e riqueza de besouros (Didham 1997b), insetos polinizadores (Aizen e Feinsinger 1994) e aranhas em ambientes alterados (Bolger et al. 2000, Gibb \& Hochuli 2002). Na Amazônia Central, alterações na dinâmica populacional das colônias de uma aranha social, Anelosimus eximius (Theridiidae), foram observadas numa floresta fragmentada de terra-firme, não sujeita a inundações periódicas (Venticinque \& Fowler 2001), porém, o conhecimento dos impactos da fragmentação de hábitats sobre a araneofauna, em geral, é escasso, principalmente, se tratando das espécies de hábitos errantes.

As aranhas das famílias Ctenidae Keyserling 1877 e Sparassidae Bertkau 1872 estão amplamente distribuídas na região amazônica e podem ser facilmente encontradas forrageando na vegetação (Höfer \& Brescovit 2001, Brescovit et al. 2003). Muitas delas forrageiam com maior freqüência no folhiço, como as espécies dos gêneros Ctenus, Centroctenus, Ancylometes e Phoneutria (Ctenidae) e Olios (Sparassidae) (Höfer et al. 1994, Gasnier \& Höfer 2001). Estas aranhas são errantes e capturam presas esperando a aproximação de suas vítimas para em seguida subjugá-las após um rápido ataque. Os esparassídeos se deslocam mais à procura de alimento (Höfer \& Brescovt 2001) e costumam ser menores que os adultos de Ctenidae, no entanto, ambos são mais ativos durante a noite e podem ser encontrados nas mesmas áreas, muitas vezes próximos uns dos outros.

A coexistência entre ctenídeos e esparassídeos sugere que eles podem estar competindo por presas, abrigos e locais para forrageamento, assim como fazem outros artrópodes de liteira (Bulltman \& Uetz 1994 e Marshall et al. 2000), portanto, é provável que estas aranhas tenham impactos negativos sobre as populações umas das outras. Além disso, a maioria das aranhas é predadora generalista (Foelix 1982) e, neste caso, a predação intraguilda também deve ser considerada como um fator limitante para o tamanho das populações (Hodge 1999).

Em uma paisagem fragmentada as mudanças na estrutura da vegetação podem afetar a abundância de invertebrados (Didham 1997a) e a ocorrência de espécies que utilizam os mesmos recursos, como no caso de abelhas, cujas dietas sobrepõe-se (Sowig 1989). Por esta razão, seria interessante determinar como a fragmentação da floresta afeta a densidade de populações, cujas espécies exploram os mesmos recursos de maneira similar.Assim, comparamos a densidade de aranhas das famílias Ctenidae e Sparassidae em fragmentos florestais e áreas não perturbadas (mata contínua) e, também, investigamos a correlação entre as suas abundâncias na área de estudo. Nosso objetivo foi determinar como a fragmentação florestal afeta a densidade destas aranhas e como a coexistência entre elas pode influenciar no tamanho destas populações em ambientes alterados.

\section{MATERIAL E MÉTODOS}

Este trabalho foi conduzido numa floresta tropical úmida de terra firme, localizada $80 \mathrm{Km}$ ao norte de Manaus, como parte do Projeto Dinâmica Biológica de Fragmentos Florestais (PDBFF). A temperatura média anual da área de estudo é de $26^{\circ} \mathrm{C}$ e a precipitação anual média é $2200 \mathrm{~mm}$ por ano, com uma estação chuvosa de Novembro a Março e uma seca de Junho a Outubro (Laurance 2001).

As aranhas foram coletadas em nove áreas de mata contínua (>1000 ha) e sete fragmentos florestais ( $\leq 10$ ha) totalizando 16 amostras $(n=16)$. Cinco amostras de mata contínua foram distribuídas em lados opostos à estrada de terra que acessa à reserva do Km 41 a, no mínimo, 400 m de distância umas das outras. As demais amostras de mata contínua estão situadas nas reservas do Cabo Frio, Florestal, Cidade Powell e na fazenda Dimona. Os fragmentos se localizam nas reservas do Colosso (uma amostra de 1 ha e outra de10 ha) e nas fazendas Dimona (duas amostras de 1 ha e uma de 10 ha) e Porto Alegre (uma amostra de 1 ha e outra 10 ha). Detalhes sobre a localização das amostras, imagens aéreas e mapas da área de estudo estão disponíveis no site http://pdbff.inpa.gov.br/area.html. Em cada amostra foram estabelecidas até oito parcelas de $60 \mathrm{~m}^{2}$ (30 x $\left.2 \mathrm{~m}\right)$ para a captura de aranhas, sendo 30 delas situadas na mata contínua e 28 nos fragmentos. A distribuição das parcelas foi feita arbitrariamente na tentativa de acessar a heterogeneidade ambiental das amostras e minimizar os efeitos de borda, cujo impacto sobre os organismos tende a ser menor longe das bordas (Laurance \& Yensen 1991).

As parcelas foram visitadas a noite, sempre após as 2100 h, e cada uma delas foi percorrida duas vezes durante uma hora. As coletas foram feitas por procura visual utilizando-se lanternas de cabeça, potes e pinças para a captura das aranhas. Como a identificação das espécies se baseia na presença da genitália, a qual está desenvolvida apenas nos adultos, este estudo foi realizado englobando as aranhas ao nível de família a fim de aproveitar todos os indivíduos coletados. As parcelas foram visitadas em duas ocasiões entre Novembro de 2001 e Maio de 2002, durante a 
estação chuvosa. Os indivíduos adultos foram depositados nas coleções de aracnídeos do Instituto Nacional de Pesquisas da Amazônia (INPA) e no Laboratório de Artrópodes do Instituto Butantan (IBSP).

A área total de coleta não foi a mesma para cada amostra. Portanto, a densidade de ctenídeos e esparassídeos em uma amostra foi expressa pela média do número de indivíduos coletados em suas parcelas. Diferenças entre fragmentos e mata contínua quanto à densidade de ctenídeos e esparassídeos foram determinadas por uma análise de variância (ANOVA). Por fim, a relação entre a densidade de aranhas das famílias Ctenidae e Sparassidae na área de estudo, foi determinada pela correlação de Pearson.

\section{RESULTADOS}

Foram coletadas 1670 aranhas da família Ctenidae das quais 19\% eram adultas. Já entre os 376 esparassídeos coletados, a presença de adultos foi de $28 \%$, portanto, não seria adequado extrapolar os efeitos da fragmentação sobre os indivíduos de ambas as famílias, baseado apenas no estudo dos poucos adultos que foram identificados (Tabela 1). Entre os ctenídeos adultos, o gênero mais coletado foi Ctenus (85\%), seguido de Centroctenus (8\%), Enoploctenus (4\%), Phoneutria (2\%) e Ancylometes (1\%). O gênero Olios (92\%) foi o mais coletado entre os esparassídeos seguido por Sampaiosia (4\%), Polybetes (3\%) e Sparassidae gen. 1 (1\%), cuja identificação não foi possível devido à ausência de revisões recentes sobre esta família (Tabela 2). A densidade de indivíduos da família Ctenidae na mata contínua (43 \pm 18 ind./parcela) foi quase o dobro da observada nos fragmentos ( $24 \pm 13$ ind./parcela, $\mathrm{F}_{1,14}=5,5$, $\mathrm{p}$ $=0,034, \mathrm{n}=16$, Fig. 1). Porém, o oposto foi observado para as aranhas da família Sparassidae, cuja densidade nos fragmentos ( $7 \pm 1$ ind./parcela) foi maior do que na mata contínua ( $4 \pm 2$ ind./parcela, $F_{1,14}=11,3, p=0,005, n=16$, Fig. 2). A correlação entre a densidade de ctenídeos e esparassídeos na área de estudo foi negativa $(\mathrm{r}=-0,527, \mathrm{n}=$ 16, Fig. 3) e, talvez, o tamanho de suas populações seja limitado pela competição por recursos ou pela predação entre estas aranhas.

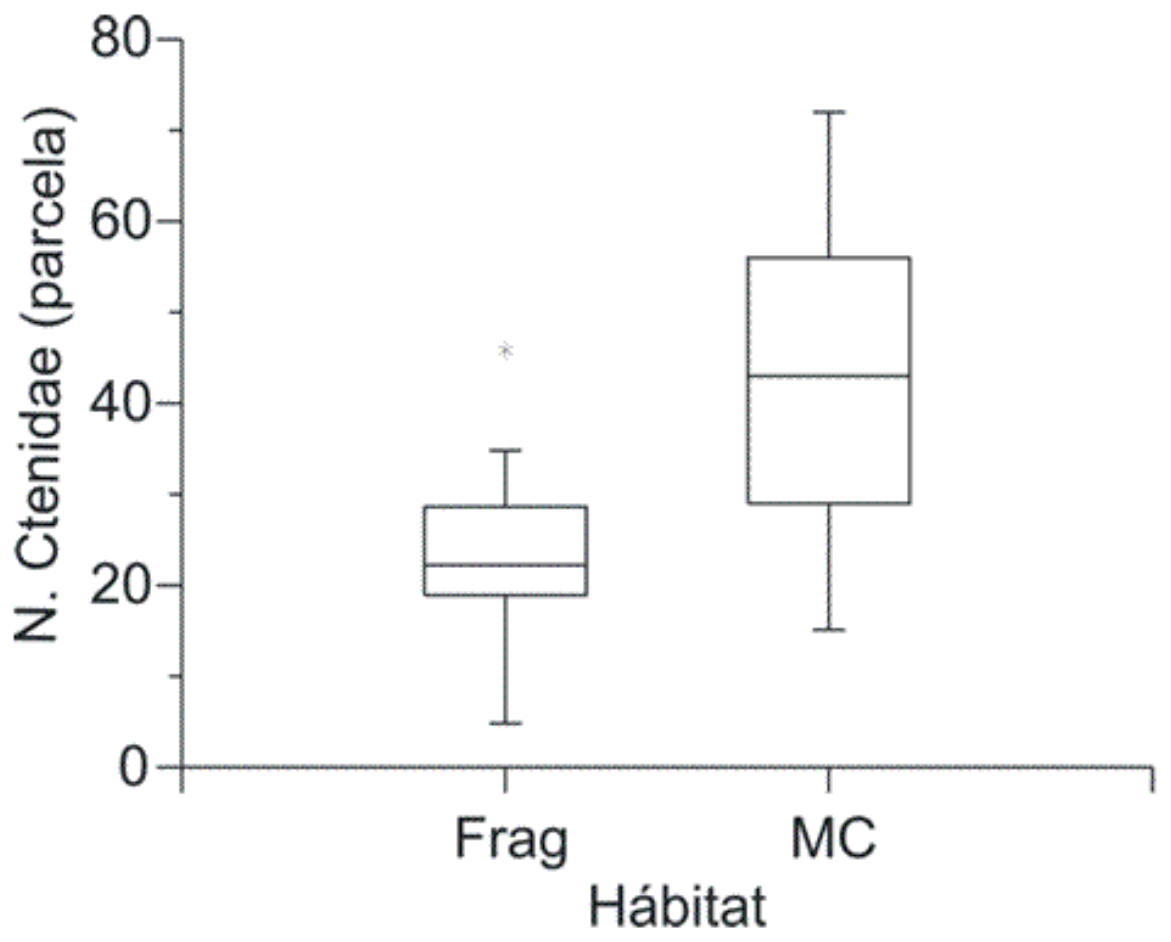

Figura 1. Densidade de aranhas da família Ctenidae. Onde, Frag = fragmentos $(\leq 10$ ha $)$ e MC = mata contínua (> 1000 ha $)$. Área da parcela $=60 \mathrm{~m}^{2}, n=16$. As linhas dentro das caixas representam a densidade média de ctenídeos em cada tratamento e o asterisco indica um valor extremo. 


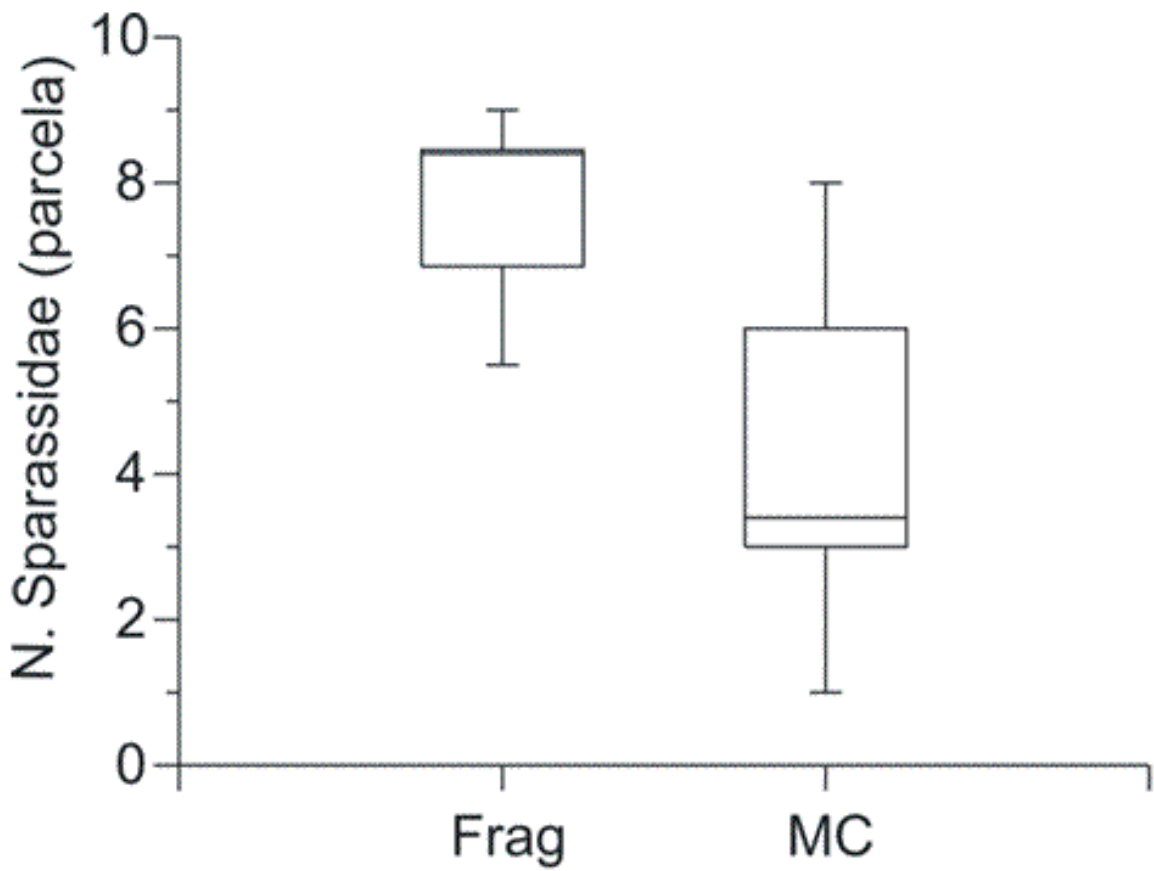

Hábitat

Figura 2. Densidade de aranhas da família Sparassidae. Onde, Frag = fragmentos $(\leq 10$ ha $)$ e MC = mata contínua $(>1000$ ha $)$. Área da parcela $=60 \mathrm{~m}^{2}, n=16$. As linhas dentro das caixas representam a densidade média de esparassídeos em cada tratamento.

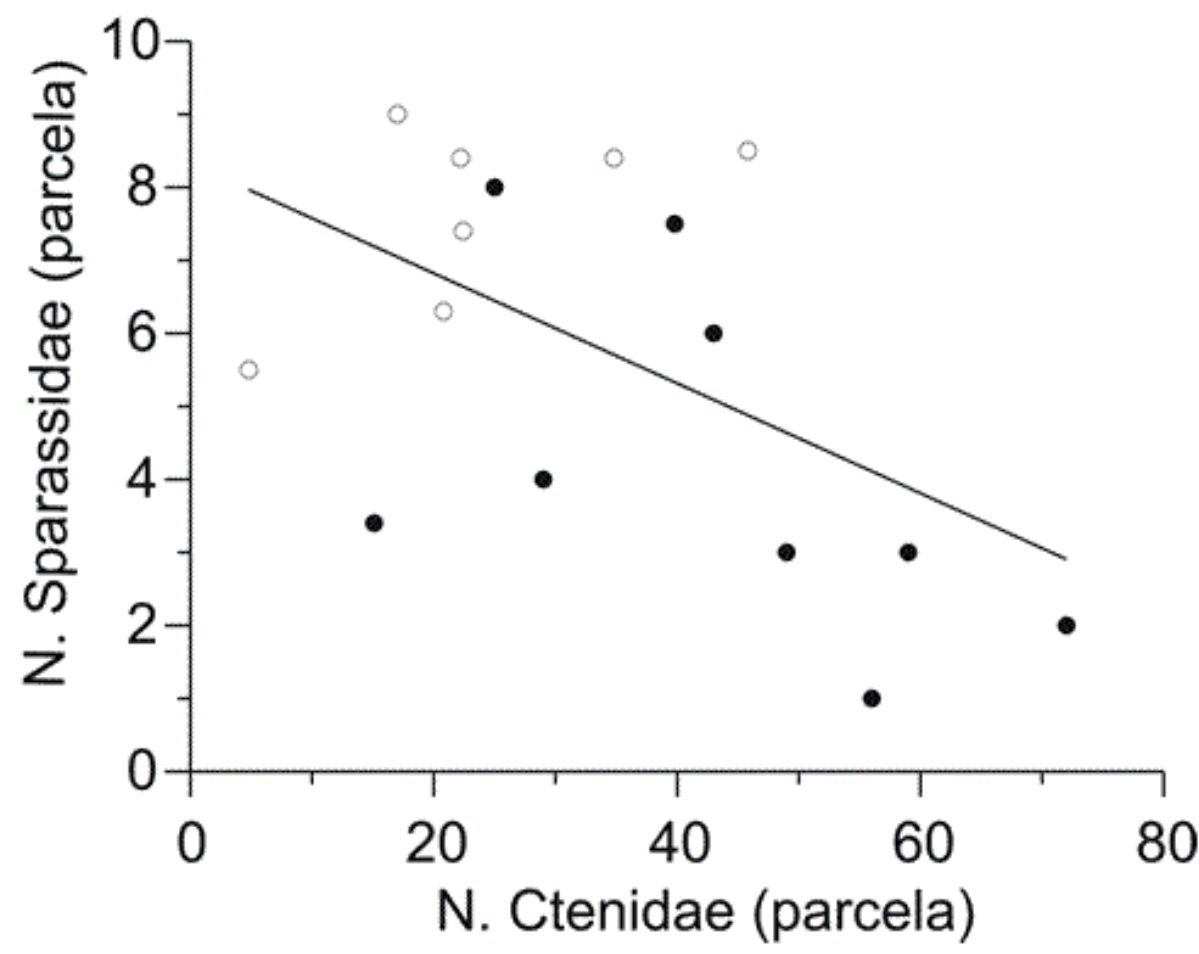

Figura 3. Correlação entre a densidade de aranhas das famílias Ctenidae e Sparassidae. Pontos escuros representam as amostras de mata contínua (>1000ha) e os pontos claros os fragmentos $(\leq 10 \mathrm{ha})$. Área da parcela $=60 \mathrm{~m}^{2}, n=16$. A correlação entre a densidade de ctenídeos e esparassídeos foi negativa na área de estudo (Pearson, $r=-0,527$ ). 


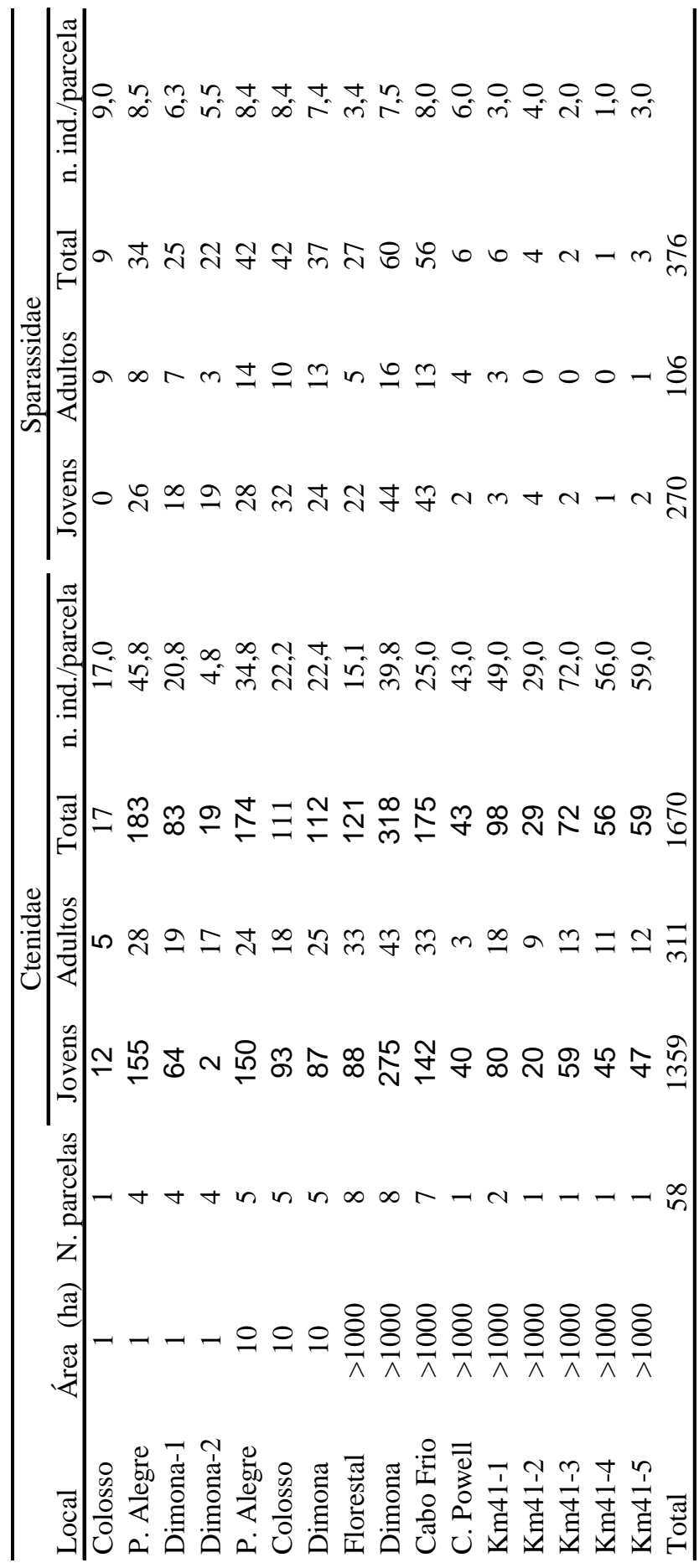




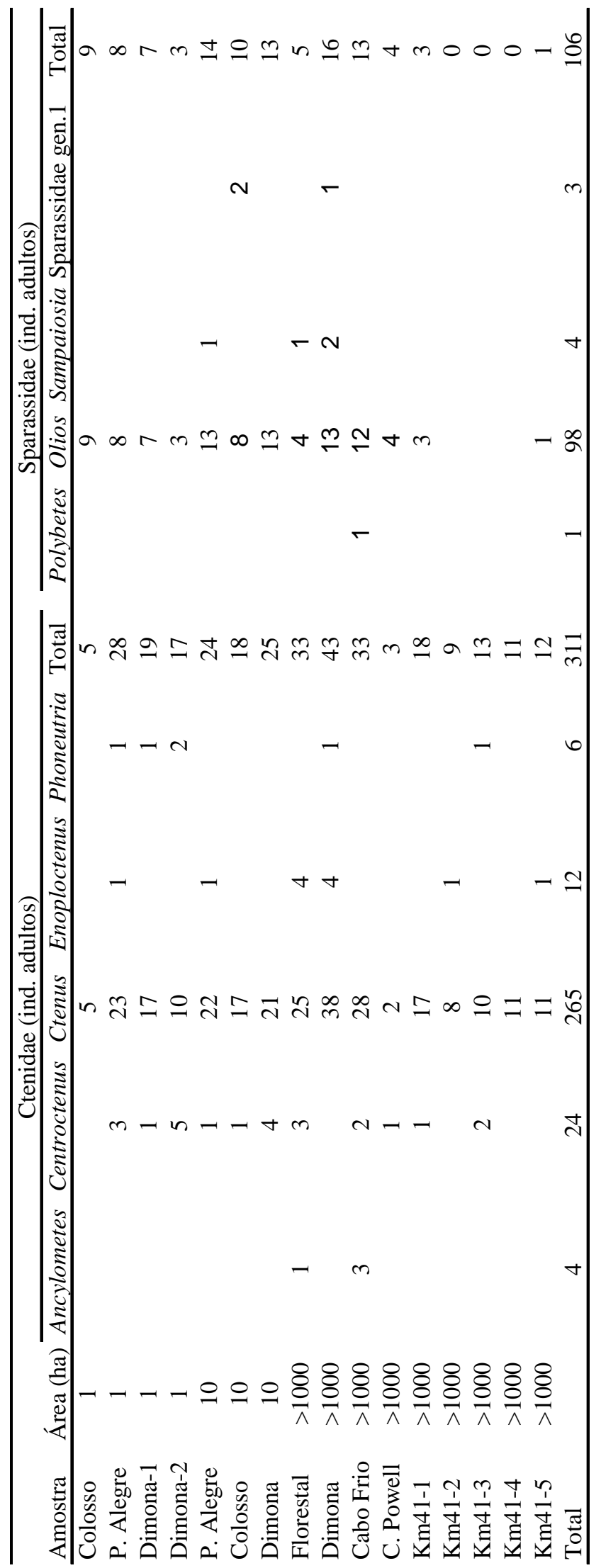




\section{DISCUSSÃO}

Ctenídeos e esparassídeos são suscetíveis ao isolamento e redução da floresta pois as densidades de suas populações nos fragmentos foram alteradas em relação ao observado na mata continua. Portanto, remanescentes de floresta primária menores que 10 ha não são capazes de manter as populações destas aranhas em níveis similares aos encontrados na mata contínua e, por esta razão, eles podem ser considerados ambientes alterados. Este argumento é reforçado por outros estudos realizados nas áreas do PDBFF, onde mudanças na composição de espécies de besouros coprófagos (Klein 1989), cupins (Souza \& Brown 1994) e borboletas (Brown \& Hutchings 1997) foram relatados em remanescentes de floresta menores que 100 ha. Além disso, a redução da floresta diminui o número de microhábitats disponíveis e a capacidade do ambiente em suportar um maior número de indivíduos (Zimmerman \& Bierregaard 1986). Porém, é necessário considerar que os fragmentos de até 10 ha têm o seu valor para a conservação da fauna de artrópodes por terem mantido a comunidade de aranhas em ambientes sujeitos a certo grau de isolamento e neste caso, este já supera 20 anos.

Aranhas da família Ctenidae foram afetadas negativamente pela fragmentação da floresta por terem suas populações reduzidas nos fragmentos. Isto sugere, que os ctenídeos provavelmente são mais exigentes quanto à qualidade do hábitat e mais vulneráveis a mudanças na estrutura na vegetação. Por outro lado, os esparassídeos parecem menos exigentes quanto á qualidade do hábitat, pois mesmo nos fragmentos, estas aranhas parecem encontrar as condições necessárias para que as suas populações se mantenham, inclusive, em maior número do que na mata contínua.

Por se tratarem de aranhas que coexistem numa mesma área, a menor densidade de ctenídeos em ambientes alterados pode ter contribuído para o aumento de esparassídeos devido a menor competição por alimento e presas. A disputa por recursos é comum na natureza (Schoener 1985) e, é possível que nos fragmentos, os esparassídeos tenham mais oportunidades em ocupar hábitats anteriormente dominados e explorados pelos ctenídeos. Experimentos com cracas (Crustacea) já demonstraram que após exclusão de uma espécie é possível que os hábitats deixados vagos por ela sejam ocupados por seus competidores (Connel 1961).

Outra hipótese a ser considerada é a redução da predação intraguilda devido a menor densidade das espécies de Ctenidae nos fragmentos. A predação entre aranhas é comum (Wise 1993) e a menor densidade de ctenídeos também pode ter favorecido o aumento das populações de esparassídeos por elas serem menos predadas. Entre as espécies mais abundantes, as aranhas adultas do gênero Ctenus são maiores que as do gênero Olios e têm maiores chances de saírem vitoriosas caso elas se enfrentem, entretanto, isto não é válido para os jovens, pois eles podem ter o mesmo tamanho. Portanto, um melhor conhecimento a respeito das interações entre ctenídeos e esparassídeos (competição interespecífica e predação intraguilda) é necessário para elucidar como a coexistência entre eles pode afetar a suscetibilidade destas aranhas à fragmentação da floresta. Isto poderia ser investigado de maneira mais apropriada manipulando a predação entre esparassídeos e ctenídeos de forma controlada em laboratório. Outra alternativa seria a exclusão de uma das espécies para avaliar se a outra passaria a ocupar os nichos vagos como prova da competição interespecífica entre elas (Wise 1981).

Neste estudo apresentamos evidências de como o isolamento e a redução da floresta afetam a densidade de ctenídeos e esparassídeos e, levantamos hipóteses sobre como a coexistência entre eles pode afetar a ocorrência destas aranhas em ambientes fragmentados. Assim, procuramos contribuir para o conhecimento dos impactos da fragmentação de hábitats sobre a fauna de artrópodes, a fim de que grandes áreas de floresta sejam preservadas para a manutenção da diversidade de espécies na Amazônia.

\section{AGRADECIMENTOS}

Agradecemos à nossa colega Cristina A. Rheims pela identificação dos exemplares de Sparassidae, ao Sr. Antonio Cardoso pela sua ajuda durante as coletas e aos dois revisores anônimos deste manuscrito pelos comentários e sugestões. Este trabalho foi financiado pelo Conselho Nacional de Desenvolvimento Científico e Tecnológico (CNPq) (processo 130083/01-1), Projeto Dinâmica Biológica de Fragmentos Florestais (PDBFF) e Fundação de Amparo à Pesquisa do Estado de São Paulo (FAPESP processo 99/05446-8). Esta é a publicação número 434 da série técnica do PDBFF.

\section{REFERÊNCIAS BIBLIOGRÁFICAS}

AIZEN, M. A. \& FEISINGER, P. 1994. Forest fragmentation, pollination, and plant reproduction in a Chaco Dry Forest, Argentina. Ecology, 75(2): 330-351.

BOLGER, D.T., SUAREZ, A.V., CROOKS, K.R., MORRISON, S.A. \& CASE, T.J. 2000. Arthropods in urban habitat fragmentation in southern California: area, age, and edge effects. Ecol. Appl. 10(4): 1230-1248.

BRESCOVIT, A. D., BONALDO, A.B., BERTANI, R. \& RHEIMS, C.A. 2003. Araneae. In Amazonian Arachnida and Myriapoda (J. Adis, ed.). Pensoft Publishers, Sofia-Moscow, p. 303-343.

BROWN, K. S. \& HUTCHINGS, R.W. 1997. Disturbance, fragmentation, and the dynamics of diversity Amazonian butterflies. In Tropical Forest Remnants: Ecology, Management, and Conservation of Fragmented Communities (W. F. Laurance \& R. O. Bierregaard, eds.), University of Chicago Press, Chicago, p. 91-110.

BULLTMAN, T.L. \& UETZ, G.W. 1984. Effect of structure and nutritional quality of litter on abundances of litter-dwelling arthropods. Am. Midl. Nat. 111(1): 165-172. 
CAMARGO, J.L.C. \& KAPOS, V. 1995. Complex edge effects on soil moisture and microclimate in Central Amazonian forest. J. Trop. Ecol. 11: 205-221.

CARVALHO, K.S. \& VASCONCELOS, H.L. 1999. Forest fragmentation in Central Amazonia and its effects on litter-dwelling ants. Biol. Conserv. 91:151-158.

CONNEL, J. H. 1961. The influence of interspecific copetition and other factors on the distribution of the barnacle Chthamalus stellatus. Ecology 42(4):710-723.

DAVIDSON, C. 1998. Issues in measuring landscape fragmentation. Wildl. Soc. Bull. 26(1):32-37.

DIDHAM, R. K. 1997a. The influence of edge effects and forest fragmentation on leaf litter invertebrates in central Amazonia. In Tropical Forest Remnants: Ecology, Management, and Conservation of Fragmented Communities (W.F. Laurance \& R.O. Bierregaard, eds.), University of Chicago Press, Chicago, p. 55-70.

DIDHAM, R.K. 1997b. An overview of invertebrate responses to forest fragmentation. In Forest and Insects (A. D. Watt, N. E. Stork \& M. D. Hunter eds.), Chapman and Hall, London, p. 301-318.

DIDHAM, R.K. \& LAWTON, J.H. 1999. Edge structure determines the magnitude of changes in microclimate and vegetation structure in tropical forest fragments. Biotropica 31(1): 17-30.

FOELIX, R.F. 1982. Biology of Spiders. Harvard University Press, London, England.

GASNIER, T. R. \& HÖFER, H. 2001. Patterns of abundance of four species of wandering spiders (Ctenidae, Ctenus) in a forest in central Amazonia. J. Arachnol. 29:95-103.

GIBB, H. \& HOCHULI, D. F. 2002. Habitat fragmentation in an urban environment: large and small fragments support different arthropod assemblages. Biol. Conserv. 106:91-100.

HODGE, M. A. 1999. The implications of intraguild predation for the role of spiders in biological control. J. Arachnol. 27: 351-362.

HÖFER, H., BRESCOVIT, A. D. \& GASNIER, T. 1994. The wandering spiders of genus Ctenus (Ctenidae, Araneae) of Reserva Ducke, a rainforest in central Amazonia. Andrias 13:81-89.

HÖFER, H. \& BRESCOVIT, A. D. 2001. Species and guild struture of Neotropical spider assemblage (Araneae) from Reserva Ducke, Amazonas, Brasil. Andrias 15:99-119.

KLEIN, B. C. 1989. The effects of forest fragmentation on dung and carrion beetle (Scarabaeinae) communities in Central Amazonia. Ecology 70(6): 17151725.

LAURANCE, W. F. The hyper-diverse flora of the Central Amazon: an overview. 2001. In Lessons From Amazonia: The Ecology and Conservation of a Fragmented Forest (R. O. Bierregaard, C. Gascon, T. E. Lovejoy \& R.. Mesquita, eds.). Yale Universit Press, New Haven, p. 47-53.

LAURANCE, W. F. \& YENSEN, E. 1991. Predicting the impacts of edge effects in fragmented habitats. Biol. Conserv. 55: 77-92.

LAURANCE, W.F., LOVEJOY, T.E., VASCONCELOS, H.L., BRUNA, E.M., DIDHAM, R.K., STOUFFER, P.C., GASCON, C., BIERREGAARD, R.O., LAURANCE, S.G. \& SAMPAIO, E. 2002. Ecosystem decay of Amazonian forest fragments: a 22-year investigation. Conserv. Biol. 16(3): 605-618.
MARSHALL, S. D., WALKER, S. E. \& RYPSTRA, A. L. 2000. A test for a differential colonization and competitive ability in two generalist predators. Ecology 81: 3341-3349.

SCHOENER, T. W. 1985. On the degree of consistency expected when different methods are used to estimate competition coefficients. Oecologia 67: 591-592.

SOUZA, O. F. F. \& BROWN, V. K. 1994. Effects of habitat fragmentation on Amazonian termite communities. J. Trop. Ecol. 10: 197-206.

SOWIG, P., 1989. Effects of flowering plant's patch size on species composition of pollinator communities, foraging strategies, and resource partitioning in bumblebees (Hymenoptera: Apidae). Oecologia 78: 550-558.

VENTICINQUE, E. M., \& FOWLER, H.G. 2001. Local Extinction Risks and Asynchronies: The evidence for a Metapopulation Dynamics of a Social Spider, Anelosimus eximius (Araneae, Theridiidae). In Lessons From Amazonia: The Ecology and Conservation of a Fragmented Forest (R. O. Bierregaard, C. Gascon, T. E. Lovejoy \& R. Mesquita, eds.). Yale Universit Press, New Haven, p. 187-198.

WISE, D. H. 1981. A removal experiment with darkling beetles: lack of evidence for interspecific competition. Ecology 62: 727738.

WISE, D. H. 1993. Spider in ecological webs. Cambridge University Press, New York.

ZIMMERMAN, B. L. \& . BIERREGAARD, R. O. 1986. Relevance of the equilibrium theory of island biogeography with an example from Amazonia. J. Biogeogr. 13: 133-143.

Título: Densidades de Aranhas Errantes (Ctenidae e Sparassidae, Araneae) em uma Floresta Fragmentada.

Autores: Felipe N. A. A. Rego, Eduardo M. Venticinque \& Antonio D. Brescovit

Biota Neotropica, Vol. 5 ( número 1a): 2005

http://www.biotaneotropica.org.br/v5n1a/pt/ abstract?article+BN002051a2005

Recebido em 07/12/2003 - Aceito em 01/02/2005

ISSN 1676-0603 